\title{
Fluorescent Ligands on the Basis of Hongotoxin 1: eGFP-Hongotoxin 1
}

Maria V. Savelieva ${ }^{1,2 *}$, Kseniya Kudryashova ${ }^{2,3}$, Oksana V. Nekrasova ${ }^{3}$ and Alexey V. Feofanov ${ }^{2,3}$

1. Biological Faculty, Shenzhen MSU-BIT University, Shenzhen, China.

2. Biological Faculty, Lomonosov Moscow State University, Moscow, Russia.

3. Shemyakin-Ovchinnikov Institute of Bioorganic Chemistry, Russian Academy of Sciences, Moscow, Russia.

* Corresponding author: maria.save1995@gmail.com

Since the early 2000s, when fluorescent proteins (FP) were functionally expressed in heterologous cells, they found a wealth of applications in molecular biology from live cell imaging to measuring proteinprotein interactions. Recently, a novel approach was developed to use FPs for labeling peptide toxins from scorpion venom, which are indispensable tools to study structure and pharmacology of potassium voltage-gated $(\mathrm{Kv})$ channels [1]. Production of genetically encoded fluorescent protein-scorpion toxin chimeras (FP-Tx) has certain advantages over synthetic labeling of peptides, namely, the targeted incorporation of FP into the N- or C-terminus of a polypeptide chain, and a simple and reproducible purification procedure, which is characterized by high yield and homogeneity of recombinant FP-Tx.

Here we used enhanced green fluorescent protein (eGFP) to tag peptide toxin hongotoxin 1 (HgTx1), which is a high-affinity blocker of a number of related Kv1 channels (Kv1.1-Kv1.3). The gene encoding eGFP-HgTx 1 was cloned into NcoI/HindIII sites of pET23d vector (Novagen) and expressed in E. coli Rosetta-gami pLysS. His6-tagged eGFP-HgTx1 protein was affinity-purified from bacteria with a high yield (about $80 \mathrm{mg}$ per 11 of culture). Absorption spectrum of obtained FP-Tx was similar to that of eGFP. To study affinity of eGFP-HgTxl to Kv1.1- and Kv1.3-binding sites, we used confocal fluorescent microscopy and bioengineered analytical systems, which are based on receptor KcsA-Kv1.x $(\mathrm{x}=1,3)$ chimeric proteins embedded in the plasma membrane of E.coli spheroplasts $[2,3]$. eGFPHgTx1 was shown to bind to KcsA-Kv1.1- and KcsA-Kv1.3-presenting spheroplasts and easily displaced from the binding sites with non-labeled HgTx1 used in several-fold excess (Fig. 1). To determine affinity of eGFP-HgTx1 to the target receptors, saturation binding curves were measured (Fig. $1 \mathrm{~B}$ ), and dissociation constants $\left(\mathrm{K}_{\mathrm{d}}\right)$ of ligand-receptor complexes were estimated: $3.2 \pm 1.4 \mathrm{nM}$ for KcsA-Kv1.1 and $1.1 \pm 0.2 \mathrm{nM}$ for KcsA-Kv1.3. The $\mathrm{K}_{\mathrm{d}}$ values correlate qualitatively with those obtained for ${ }^{125} \mathrm{I}-\mathrm{HgTX} 1-\mathrm{A} 19 \mathrm{Y} / \mathrm{Y} 37 \mathrm{~F}$ in a radioligand assay $(0.4 \mathrm{nM}$ for KcsA-Kv1.1 and $0.03 \mathrm{nM}$ for KcsAKv1.3) [4].

We carried out detailed studies to characterize ability of different Kv1-channel blockers belonging to $\alpha$ KTx family of scorpion venom - derived peptides to displace eGFP-HgTx1 from the complexes with KcsA-Kv1.3 in a competitive binding assay (Fig.1 C). For this, peptide toxins HgTx1, agitoxin 2 (AgTx2), kaliotoxin (KTX), and charybdotoxin (ChTx), which were obtained in a recombinant form according to the previously developed technique [5], were used. All these peptides exhibited high affinity to the receptor KcsA-Kv1.3 protein, and apparent dissociation constants $\left(\mathrm{K}_{\mathrm{i}}\right)$ were determined to be: $200 \mathrm{pM}$ for HgTx1, $245 \mathrm{pM}$ for AgTx2, $115 \mathrm{pM}$ for ChTx, and $20 \mathrm{pM}$ for KTX. These $\mathrm{K}_{\mathrm{i}}$ values are similar to the known ones estimated for the binding of these toxins to the eukaryotic Kv1.3 channel. These results suggest that binding of eGFP-HgTx1 to the target KcsA-Kv1.3 channel is specific and reversible, and eGFP-HgTx1 fluorescent protein chimera competes with venom toxins for the same binding site of KcsA-Kv1.3. Thus, adding a bulky protein molecule, eGFP, to the peptide toxin does not 
perturb its interaction with the receptor binding site. The obtained eGFP-HgTx1 chimeric protein is a prospective fluorescent probe for the screening of Kv1.3-channel ligands [6].

References:

[1] AI Kuzmenkov et al., Sci. Rep. 6 (2016), p. 33314.

[2] AI Kuzmenkov et al., J Biol. Chem. 290 (2015), p. 12195-12209.

[3] KS Kudryashova et al., Anal. Bioanal. Chem. 405 (2013), p. 2379-2389.

[4] C Legros et al., Biochemistry 41 (2002) p.15369-15375.

[5] O Nekrasova et al., J. Biotechnol. 241 (2017) p.127-135.

[6] Financial support of the grant of Presidium RAS is acknowledged.
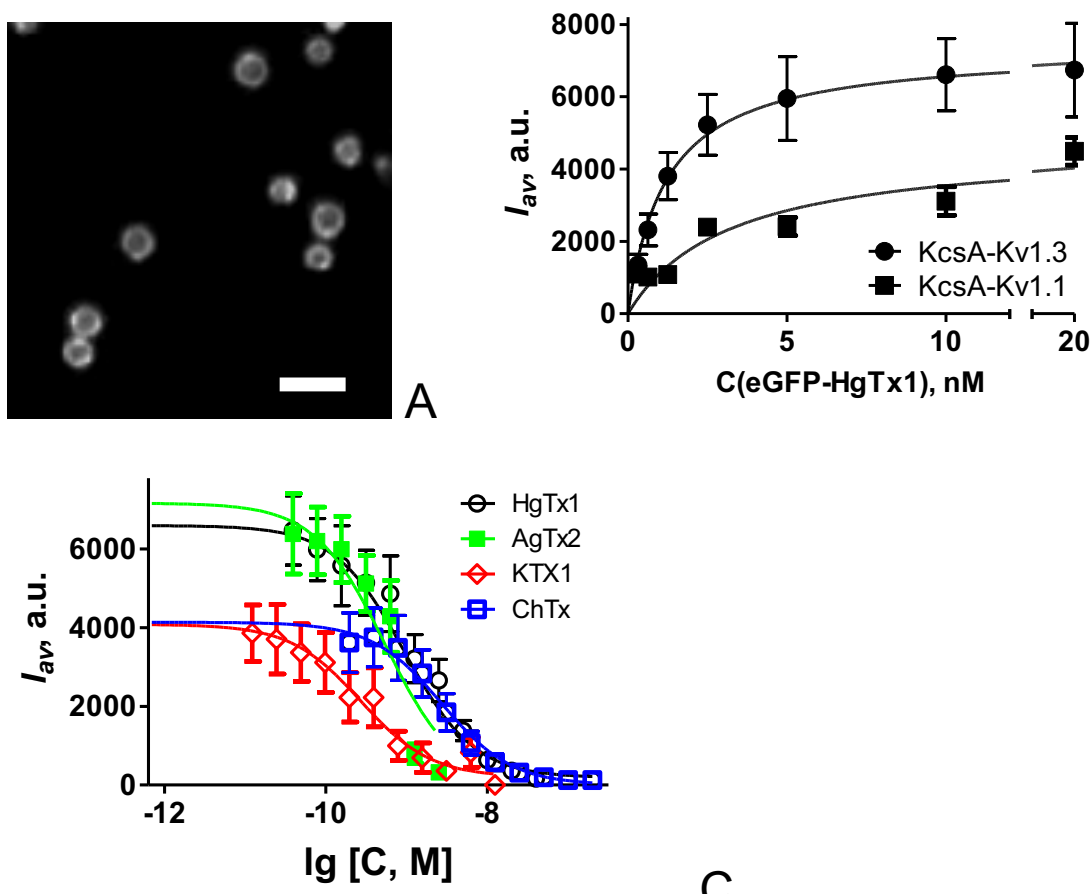

Figure 1. Interactions of eGFP-HgTx1 with spheroplasts bearing KcsA-Kv1.x (x=1, 3) hybrid channels. (A) A confocal fluorescence image showing staining of KcsA-Kv1.3-bearing spheroplasts with eGFPHgTx1. Bar is $3 \mu \mathrm{m}$. (B) Concentration dependent binding of eGFP-HgTx1 to spheroplasts bearing KcsA-Kv1.x $(\mathrm{x}=1,3)$. (C) Competition between eGFP-HgTx1 and different ligands for the binding to KcsA-Kv1.3-presenting spheroplasts. 